\title{
Ueber einen phosphorhaltigen Bestandtheil der Pflanzensamen. von
}

E. Schnize und F. Winterstein.

(Aus dem agricultur-chemischen Laboratorium des Polytechnikums in Zürich.) (Der Redaction zugegangen am 28. Mārz 1896.)

Bei der Darstellung von Eiweiss aus Pflanzensamen erhielt W. Palladin ${ }^{1}$ ) als Nebenproduct eine Substanz, die sich in Folge ihres eigenthümlichen Verhaltens gegen Lösungs-. mittel isoliren liess. Als er nämlich die bei Extraction der gepulverten Pflanzensamen mit 10 procentiger Kochsalzlösung gewonnene eiweisshaltige Flüssigkeit auf circa $80^{\circ}$ erhitzte, schied sich jene Substanz mit den Eiweissstoffen aus, ging aber beim Erkalten der Flüssigkeit wieder in Lösung; als dann das Filtrat vom Eiweisscoagulum wieder zum Kochen erhitzt wurde, schied sie sich wieder aus und konnte nun durch Abfiltriren auf einem Heisswassertrichter und darauffolgendes Auswaschen mit heisser Kochsalzlösung und heissem Wasser von den beigemengten Substanzen befreit werden.

Als der genannte Forscher im Jahre 1894 einige Wochen lang in unserem Laboratorium arbeitete, veranlasste er uns, die weitere Untersuchung dieser Substanz auszuführen. Die dabei bis jetzt gewonnenen Resultate theilen wir in Folgendem in aller Kürze mit.

Einige Versuche, die wir noch während der Anwesenheit W. Palladin's in unserem Laboratorium mit einer von ihm selbst aus den Samen von Sinapis nigra dargestellten

1) Beiträge zur Kenntniss pflanzlicher Eiw eissstoffe, Zeitschrift f. Biologie, Jahrgang 1894, S. 199. 
Probe der fraglichen Substanz ausführten, zeigte, dass dieselbe stickstofffrei ist und dass sie beim Verbrennen eine phosphorsäure- und kalkreiche Asche liefert, welche daneben aber auch Magnesia enthält, während dagegen ihre Salpetersäure-Lösung mit Molybdänsolution keine Phosphorsäurereaction gibt. Man- darf daher vermuthen, dass sie das Calcium-Magnesiumsalz einer sogenannten gepaarten Phosphorsäure ist.

Es war nun unsere nächste Aufgabe, eine grössere Quantität dieser Substanz darzustellen. Als Material verwendeten wir dazu die Samen von Sinapis nigra, die nach Palladin's Versuchen besonders reich daran sind. Ueber das Darstellungsverfahren ist Folgendes anzugeben: 2 Kilo fein zerriebener, entfetteter Samen wurden mit 15 Liter 10 procentiger Kochsalzlösung 2 Tage lang bei Zimmertemperatur digerirt, der dabei erhaltene Extract mit Hilfe von Filtrirtuch vom Ungelösten getrennt, sodann 10--15 Minuten im Sieden erhalten und nun abgekühlt.

Dann brachten wir die Flüssigkeit mit dem darin befindlichen Eiweisscoagulum auf Faltenfilter und wuschen mit kaltem Wasser aus. Das Filtrat wurde zur Entfernung von Eiweissresten nochmals zum Sieden erhitzt, wieder abgekühlt und durch darauffolgende Filtration von der geringen Ausscheidung getrennt. Die so erhaltene Flüssigkeit trübte sich stark beim Erhitzen, wurde aber beim Erkalten wieder völlig klar, woraus hervorgeht, dass sie keine coagulirbaren Eiweissstoffe mehr enthielt. Wir erhitzten sie nun wieder zum Kochen, brachten die dabei entstandene Ausscheidung auf ein Faltenfilter, das sich auf einem durch Wasserdampf erhitzten Trichter befand; nachdem die Flüssigkeit abgeflossen war, wurde der Filterinhalt mit kochendem Wasser ausgewaschen. Derselbe bildete nun eine weisse amorphe Substanz, welche vom Kochsalz noch nicht völlig befreit war; in kaltem Wasser löste sie sich nicht wiøder auf. Zur vollständigen Beseitigung des Kochsalzes wurde solange mit destillirtem Wasser ausgewaschen, bis die ablaufende Flüssigkeit mit Silbernitrat nur noch ganz schwache Opalescenz zeigte, darauf wurde successive mit 95 procentigem 
Alkohol, absolutem Alkohol und Aether gewaschen und über Schwefelsäure im Exsiccator getrocknet. Aus 2 Kilo entfetteter Samen erhielten wir in dieser Weise $17 \mathrm{gr}$. der Substanz.

Es zeigte sich, dass durch einmalige Extraction der gepulverten und entfetteten Samen mit 10 procentiger Kochsalzlösung die in Rede stehende Substanz nicht vollständig extrahirt wurde; der dabei verbliebene Samenrückstand lieferte bei nochmaliger Extraction mit 10 procentiger Kochsalzlösung eine Flüssigkeit, die, in obiger Weise behandelt, noch $18 \mathrm{gr}$. der fraglichen Substanz lieferte.

Man kann zur Darstellung dieser Substanz auch einen anderen Weg einschlagen ${ }^{1}$ ). Wenn man nämlich den bei Behandlung der entfetteten Samen mit Kochsalzlösung erhaltenen Extract mit Ammoniak versetzt, so entsteht ein ziemlich starker Niederschlag; derselbe wurde abfiltrirt, nach dem Auswaschen mit verdünnter Essigsäure behandelt, die vom Ungelösten durch Filtration getrennte Flüssigkeit mit Ammoniak nahezu neutralisirt und sodann zum Kochen erhitzt; dabei entstand eine Ausscheidung, die sich beim Erkalten wieder auflöste, beim Erhitzen wieder erschien. Sie wurde mit Hilfe des Heisswassertrichters von der Flüssigkeit getrennt und ausgewaschen, doch ist es sehr schwierig, den Filterinhalt ganz vom Ammoniak zu befreien. In dieser Weise erhielten wir aus 1,8 Kilo entfetteter Samen 39 gr. Substanz.

Die in dieser Weise gewonnene Substanz bildet eine weisse, amorphe, leicht zerreibliche Masse, welche das oben angegebene Verhalten zeigt. Sie ist leicht löslich in verdünnten Mineralsäuren, sowie auch in verdünnter Essigsäure. Wenn man ihre Lösung in Essigsäure annähernd neutralisirt und sodann erhitzt, so entsteht eine starke Trübung, welche beim Erkalten der Flüssigkeit wieder verschwindet, beim Wiedererhitzen aber wieder auftritt. Dieses Verhalten ist ohne Zweifel darauf zurückzuführen, dass die fragliche Sub-

1) Nach dieser Methode hat auch A, von Planta in unserem Lahoratorium die fragliche Substanz aus den Samen von Sin a pis nigra dargestellt; die von ihm geplante Untersuchung dieser Subsianz wurde durch seinen im Februar 1895 leider erfolgten Tod unterbrochen. 
stanz in heissen Flüssigkeiten weniger löslich ist, als in kalten. Da sie aber nach der Reindarstellung in reinem Wasser und 10 procentiger Kochsalzlösung sich nicht auflöst, so muss man annehmen, dass beim Behandeln der Pflanzensamen mit Kochsalzlösung Säuren oder irgend welche andere in den Extract übergehende Nebenbestandtheile die Auflösung jener Substanz bedingen.

Die Elementaranalyse ${ }^{1}$ ) eines nach dem zuerst beschriebenen Verfahren dargestellten Präparats gab folgende Resultate:

a) 0,4152 gr. Substanz gaben 0,1480 gr. $\mathrm{CO}_{2}, 0,1060$ gr. $\mathrm{H}_{2} \mathrm{O}$ und 0,2818 gr. Asche.

b) 0,4698 gr. Substanz gaben 0,1650 gr. $\mathrm{CO}_{2}, 0,1196$ gr. $\mathrm{H}_{2} \mathrm{O}$ und 0,3190 gr. Asche.

Aus diesen Daten berechnet sich folgender C-, H- und Aschen-Gehalt:

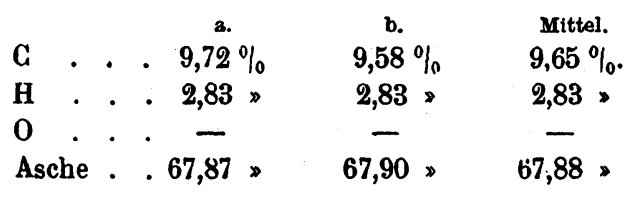

Aus dem Phosphorsäure-Gehalt einer in geeigneter Weise $^{2}$ ) dargestellten Ascheprobe würde sich für die ursprüngliche Substanz ein Gehalt von $34,66 \%, \mathrm{P}_{2} \mathrm{O}_{8}$ berechnen.

Welcher Art der in der Substanz vorhandene organische Paarling ist, vermögen wir zur Zeit noch nicht zu sagen. Versuche zur Aufklärung dieser Frage sind in unserem Laboratorium in Angriff genommen und hoffen wir, später darüberberichten zu können.

Die Substanz, auf welche sich die im Vorigen gemachten Mittheilungen beziehen, ist vielleicht identisch mit dem Hauptbestandtheil der in den Proteïnkörnern vieler Pflanzensamen als Einschlüsse sich findenden Globoide.

1) Die Substanz wurde im beiderseitig offenen Rohr im Schiffchen im Luft - resp. Sauerstoffstrom verbrannt. Die Asche war nicht vollständig weiss, sie hinterliess nach dem Auflösen in Salpetersäure aber nur Spuren von Kohle.

2) Zur Darstellung dieser Aschenprobe wurde die Substanz unter Zusatz von Soda und Salpeter verbrannt. 


\section{4}

Pfeffe $r^{1}$ ) hat solche Globoide in Verbindun mit Brandau einer Untersuchung unterworfen und dabi gefunden, dass sie aus dem Calcium-Magnesiumsalz eir mit einem organischen Körper gepaarten Phosphorsäure beehen. Welcher Art der organische Paarling ist, konnte vo den Genannten nicht festgestellt werden.

Da ausserdem in den Pflanzen bisher nur eine geaarte Phosphorsäure nachgewiesen ist, nämlich die Glyceriphosphorsäure, welche zwar nicht frei, aber doch als Bestantheil des Lecithins gefunden wurde, so darf die im Vorige beschriebene Substanz wohl Interesse beanspruchen.

1) W. Pfeffer: Untersuchung über die Proteïnkörner ud die Bedeutung des Asparagins beim Keimen der Samen. Jahrbücłr für wissenschaftliche Botanik, 8. Bd., 1872, S. 147. 